\title{
REVISTAMATĒRIA
}

ISSN 1517-7076 artículo e-12058, 2018

\section{Síntesis y caracterización de "tanatos" de lantano como inhibidores de la corrosión}

\author{
Oriana D’Alessandro ${ }^{1,2}$, Gonzalo Julián Selmi ${ }^{2}$, \\ Cecilia Deyá ${ }^{2,3}$, Roberto Romagnoli ${ }^{2,3}$
}

\footnotetext{
${ }^{1}$ Departamento de Química, Facultad de Ciencias Exactas, Universidad Nacional de La Plata, 47 y $115 \mathrm{~s} / \mathrm{n}^{\circ}$ La Plata, Buenos Aires, Argentina, e-mail: oriana@quimica.unlp.edu.ar ${ }^{2}$ CIDEPINT-CICPBA-CONICET, Av. 52 e/ 121 y 122,sin n ${ }^{\circ}$, La Plata, Buenos Aires, Argentina.

${ }^{3}$ Departamento de Ingeniería Química, Facultad de Ingeniería, Universidad Nacional de La Plata, 1 y $47 \mathrm{~s} / \mathrm{n}^{\circ}$ La Plata, Buenos Aires, Argentina.
}

\section{RESUMEN}

Los taninos son polifenoles sintetizados por las plantas. Se encuentran en la madera del Quebracho (Schinopsis balansae), los frutos de la Tara (Caesalpinia spinosa), etc. Han sido incorporados en la industria de la pintura como agentes "antifouling" y como inhibidores de la corrosión en soluciones ácidas. Por otra parte, las soluciones de lantano han presentado un efecto inhibidor muy satisfactorio sobre probetas de acero SAE 1010.

El objetivo del presente trabajo fue el de obtener dos tipos de "tanato" de lantano de baja solubilidad, a fin de que puedan ser incorporados como inhibidores de la corrosión en recubrimientos protectores temporarios, que protegen el metal durante su traslado y almacenamiento y, eventualmente, en pinturas anticorrosivas. En este trabajo se presenta la obtención de los "tanatos", su caracterización y ensayos electroquímicos en solución de $\mathrm{NaCl}$. Se prevé que si estos ensayos son satisfactorios, los "tanatos" puedan ser incorporados en protectores temporarios.

Se obtuvieron 2 "tanatos", empleando taninos de tara y de quebracho comerciales y solución de $\mathrm{La}\left(\mathrm{NO}_{3}\right)_{3} \times 6 \mathrm{H}_{2} \mathrm{O} \quad 0,1 \mathrm{M}$. Los pigmentos se caracterizaron mediante espectrometría infrarroja con transformada de Fourier (FTIR) y su solubilidad fue determinada mediante una técnica colorimétrica utilizando el reactivo de Folin-Denis, mientras que el $\mathrm{La}^{+3}$ soluble se determinó por una técnica gravimétrica con oxalato. La acción protectora de los "tanatos" sintetizados, frente a la corrosión del acero SAE 1010 fue evaluada mediante curvas de polarización y medidas de potencial de corrosión en solución de $\mathrm{NaCl}$. La película protectora formada sobre el acero inmerso en la solución fue observada mediante microscopía electrónica de barrido (MEB) y caracterizada mediante espectroscopía de dispersión de rayos X (EDS).

Ambos "tanatos" desplazaron el potencial de corrosión del acero hacia valores más positivos y la resistencia a la polarización resultó uno o dos órdenes de magnitud mayor, con respecto al blanco (solución sin inhibidor) y a soluciones que contenían el tanino comercial correspondiente en diferentes concentraciones. Por otro lado, las determinaciones de la solubilidad de los pigmentos hacen preveer que no serán lixiviados de los recubrimientos prematuramente y que podrán ejercer su acción protectora durante un tiempo adecuado.

Palabras clave: taninos, lantano, medidas electroquímicas, corrosión.

\section{ABSTRACT}

Tannins are polyphenols synthesized by plants. They are found in Quebracho (Schinopsis Balansae) wood, Tara (Caesalpinia spinosa) fruits, etc. They have been incorporated in the coating industry as "antifouling" agents and as corrosion inhibitors in acidic solutions. Moreover, lanthanum solutions have a great inhibitory effect over SAE 1010 steel corrosion.

The aim of this study was to obtain two types of lanthanum "tannate" with low solubility, so they can be incorporated as corrosion inhibitors in temporary protective coatings, those that protect the metal during transportation and storage. Eventually, they could also be employed in anticorrosive paints. 
Two types of "tannates" were obtained with two commercial Tara and Quebracho tannins and 0.1M $\mathrm{La}\left(\mathrm{NO}_{3}\right)_{3} \mathrm{x} 6 \mathrm{H}_{2} \mathrm{O}$. The pigments were characterized by Fourrier transformance infra-red spectroscopy (FTIR) and their solubility was determined by the Folin-Denis technique. The protective action of "tanates" on SAE 1010 steel was evaluated by polarization curves and corrosion measurements in $\mathrm{NaCl}$ solution. The protective film formed on the steel immersed in the "tannate" suspension was observed by scanning electron microscopy and characterized by dispersive X-ray spectroscopy. Both "tannates" shifted the corrosion potential to more positive values and the polarization resistances were one or two orders of magnitude higher than the controls (solution without inhibitor and different concentration of commercial tannin solutions). On the other hand, the quantification of soluble metal cation and oxidizable material suggests that no premature lixiviation will occur from the coating and they can perform their protective properties for a suitable time.

Keywords: tannins, lanthanum, electrochemical measurements, corrosion.

\section{INTRODUCCIÓN}

Los recubrimientos protectores temporarios llamados "wash primers" o imprimaciones de lavado, protegen las superficies metálicas durante la etapa de almacenamiento y/o transporte de las piezas previo al pintado. Se trata de mezclas fluidas que generan una película protectora por evaporación del disolvente. Estas formulaciones contienen inhibidores de corrosión tradicionales, como el tetroxicromato de zinc, los cuales por resultar tóxicos deben ser reemplazados. En este sentido, la búsqueda se orientó al estudio de nuevos pigmentos de origen vegetal, los cuales resultan amigables con el medioambiente.

Los taninos son compuestos polifenólicos y pertenecen a los metabolitos secundarios de las plantas. Son sustancias que no parecen tener una función directa en procesos fotosintéticos, respiratorios, de asimilación de nutrientes, etc, sino que intervienen en las interacciones entre la planta y su ambiente, tienen la capacidad de unirse por puente de hidrógeno a proteínas y formar complejos indigeribles, por lo cual actúan como repelentes alimenticios de muchos herbívoros. Han sido incorporados en la industria de la pintura como agentes "antifouling" [1] y como inhibidores de la corrosión en soluciones ácidas [2, 3, 4]. La capacidad pasivante de algunos taninos por reacción química directa con el sustrato metálico oxidado ha sido reportada desde los años ' 70 [2, 5, 6].

Por otro lado, el lantano es un elemento de las tierras raras, debido a su relativa escasez en la corteza terrestre. No se presenta en estado elemental sino que principalmente se encuentra en la "monacita", un silicato que contiene fósforo y una cantidad variable de otros lantánidos. Se puede adquirir comercialmente como sales de $\mathrm{La}$ (III) por ej.: $\mathrm{La}\left(\mathrm{NO}_{3}\right)_{3} \times 6 \mathrm{H}_{2} \mathrm{O}$. La capacidad pasivante del catión $\mathrm{La}$ (III) se ha caracterizado a través de medidas electroquímicas, entre otras [7].

Teniendo en cuenta lo dicho anteriormente, se prepararon y evaluaron pigmentos a base de tanino de Quebracho (Schinopsis balansae), tanino de Tara (Caesalpinia spinosa) y sus respectivas sales de lantano, precipitando los correspondientes "tanatos" metálicos. Luego, se ensayaron los pigmentos como inhibidores de la corrosión de acero SAE 1010 en solución de $\mathrm{NaCl}$.

En este trabajo se presenta un estudio comparativo entre dos "tanatos" precipitados con La(III) cuya diferencia es la matriz orgánica utilizada (tanino de Tara y tanino de Quebracho), para de esta manera analizar el efecto sinérgico entre el La y el tanino.

\section{MATERIALES Y MÉTODOS}

\subsection{Síntesis de "tanatos" de Quebracho y de Tara}

Los taninos solubles se obtuvieron por extracción en caliente con agua destilada a partir de los taninos sólidos comerciales [8] y los "tanatos" por precipitación de los antes mencionados con una sal de lantano a pH controlado. Se emplearon taninos de quebracho (UNITAN) y de tara (INDUNOR).

A partir de los taninos comerciales de Quebracho y de Tara se prepararon soluciones madre de taninos, dispersando los taninos comerciales en agua destilada a $90^{\circ} \mathrm{C}$ durante 1 hora, filtrando al vacío y llevando a $1000 \mathrm{ml}$ con agua destilada.

Luego, distintas porciones de las soluciones anteriores se pusieron en contacto con $\mathrm{La}\left(\mathrm{NO}_{3}\right)_{3} \times 6 \mathrm{H}_{2} \mathrm{O}$ (Merck) $0,1 \mathrm{M}$ ajustando el pH a 8 con $\mathrm{NaOH} 1 \mathrm{M}$, con agitación continua durante $1 \mathrm{~h}$, el precipitado obtenido en cada caso se lavó con agua destilada y se dejó secar a $100{ }^{\circ} \mathrm{C}$ en estufa durante 24 h. De esta manera se construyeron las curvas de precipitación de los "tanatos", que permitieron seleccionar las respectivas rutas de síntesis de los pigmentos en cuestión (Figura 1). Se obtuvieron así el "tanato 
de Quebracho" (TANLA Q) y el “tanato de Tara” (TANLA T).

\subsection{Caracterización de taninos y "tanatos"}

Los pigmentos se caracterizaron mediante espectroscopía FTIR y Difracción de Rayos X (DRX). Para obtener la solubilidad de los mismos, se determinó el contenido de polifenoles totales mediante el reactivo Folin-Denis [9] y de La(III) por precipitación con oxalato de sodio 1N [10].

\subsection{Estudio de la capacidad inhibidora de corrosión de los pigmentos}

Los potenciales de corrosión en función del tiempo se midieron respecto al electrodo de calomel saturado (ECS), sumergiendo un panel de acero SAE 1010 (área expuesta de $1 \mathrm{~cm}^{2}$ ) en soluciones de los taninos, de $\mathrm{La}\left(\mathrm{NO}_{3}\right)_{3} \times 6 \mathrm{H}_{2} \mathrm{O}$ y en suspensiones de los pigmentos. Como medio agresivo se empleó solución de ClNa $0,025 \mathrm{M}$.

La velocidades de corrosión (Jcorr) en suspensiones de los taninos y "tanatos" se obtuvieron sumergiendo electrodos de acero SAE 1010, con un área expuesta de $0,28 \mathrm{~cm}^{2}$, en ClNa $0,1 \mathrm{M}$ como medio agresivo a una velocidad de barrido de $0,5 \mathrm{mV} / \mathrm{seg}$ con una amplitud de $\pm 0,030 \mathrm{~V}$ o.c. utilizando un ECS como referencia y un contraelectrodo de Pt. Como control se utilizó acero SAE 1010 inmerso en las soluciones respectivas de $\mathrm{NaCl}$.

El cálculo de Jcorr se llevó a cabo a partir de las curvas de polarización lineal, calculando la pendiente de la recta $\delta E / \delta \mathrm{J}$ usando el software GamryChem analysis.

\subsection{Análisis de la superficie}

Luego de 24 h de inmersión en el medio agresivo, y bajo la acción de los taninos y "tanatos" respectivos, los paneles ensayados fueron analizados mediante MEB y EDS, para así determinar las características morfológicas y la composición del film protector.

\section{RESULTADOS}

\subsection{Síntesis de "tanatos" de Quebracho y de Tara}

En la Figura 1 se muestran las curvas de masa de tanato en función del volumen de solución de La(III) agragado. Se observa que luego de agregar $30 \mathrm{~mL}$ de la solución, la masa de TANLA Q o TANLA T no se ven incrementadas con lo cual fue esta relación la utilizada para obtener los respectivos "tanatos".

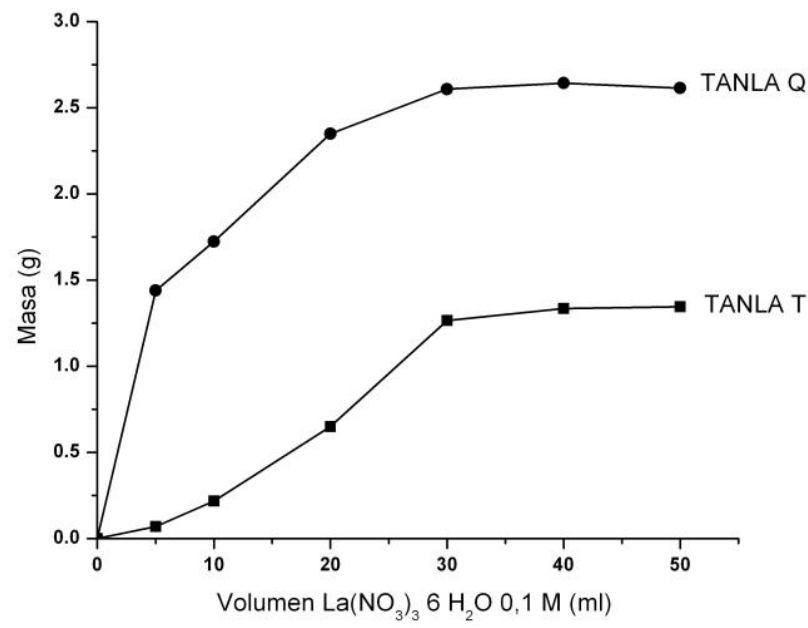

Figura 1: Masa de "tanato" vs. volumen de $\mathrm{La}\left(\mathrm{NO}_{3}\right)_{3} \times 6 \mathrm{H}_{2} \mathrm{O}$. 


\subsection{Caracterización de los "tanatos" de Quebracho y de Tara}

Mediante espectroscopía FTIR se analizaron los taninos comerciales de Quebracho (TQ) y de Tara (TT), como así también el TANLA Q y el TANLA T.

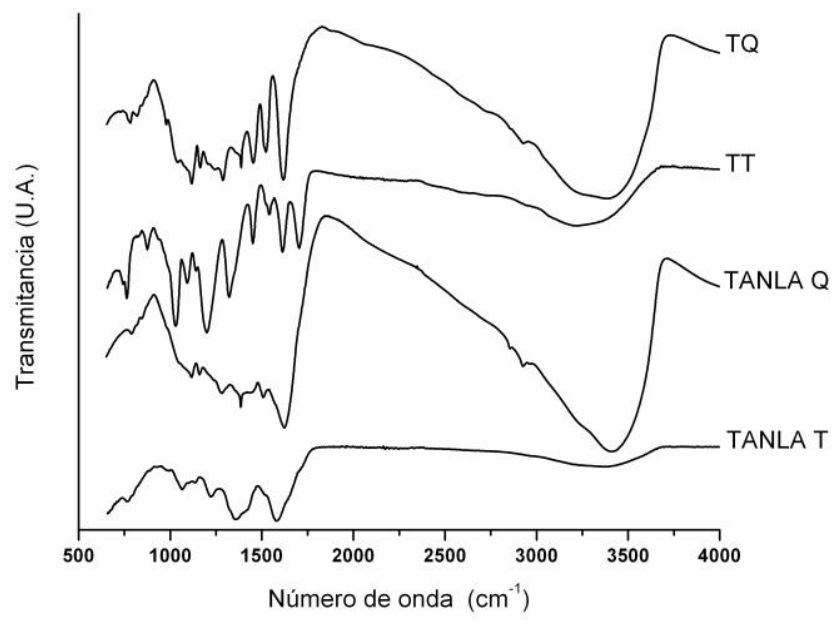

Figura 2: Espectros FTIR de los diferentes taninos (TQ, TT) y tanatos (TANLA Q, TANLA T).

En el espectro de las muestras TQ y TT se observa para la región que va desde 3600 a $3000 \mathrm{~cm}^{-1}$ una banda ancha asociada al estiramiento $-\mathrm{OH}$ proveniente de diferentes entornos químicos, lo cual es característico de los extractos polifenólicos y, si bien el pigmento fue secado hasta peso, constante puede deberse a la presencia de agua remanente. También se detectan bandas vinculadas a las vibraciones del grupo $\mathrm{C}=\mathrm{C}$ - aromático a $1619,1529,1447 \mathrm{~cm}^{-1}$ para TQ y a 1610,1537 y $1455 \mathrm{~cm}^{-1}$ para TT. Los estiramientos C-O se detectaron en $1391,1293,1160$ y $983 \mathrm{~cm}^{-1}$ para TQ y a 1318,1203 y $1024 \mathrm{~cm}^{-1}$ para TT, debiéndose las diferencias al diferente entorno químico. Las señales C-H aromáticas en el plano se ubicaron en 1122 y $1032 \mathrm{~cm}^{-1}$ para TQ y a 1138 y $1097 \mathrm{~cm}^{-1}$ para TT mientras que las bandas que corresponden C-H aromático fuera del plano se encontraron alrededor de $830 \mathrm{~cm}^{-1}$ para ambos taninos. La banda ubicada a $1709 \mathrm{~cm}^{-1}$ es el resultado del estiramiento $\mathrm{C}=\mathrm{O}$ característico de los esteres presentes en los taninos hidrolizables, especialmente los derivados del ácido gálico, la cual nos permite distinguir taninos hidrolizables como TT de taninos condensados como TQ, ya que estos últimos, en condiciones naturales, no contienen carbonilo [11].

Para las muestras TANLA Q y TANLA T no se observan las bandas de absorción $\mathrm{La}_{2} \mathrm{O}_{3}$ que normalmente se encuentran a 464 y $571 \mathrm{~cm}^{-1}$ [12], sin embargo se evidencia un cambio de estructura respecto a los taninos correspondientes, lo cual se asocia a la presencia de lantano en la matriz.

Por el análisis DRX se estudiaron sendas porciones de TANLA Q y TANLA T calcinadas a $850{ }^{\circ} \mathrm{C}$ en atmósfera de aire durante $3 \mathrm{~h}$. 


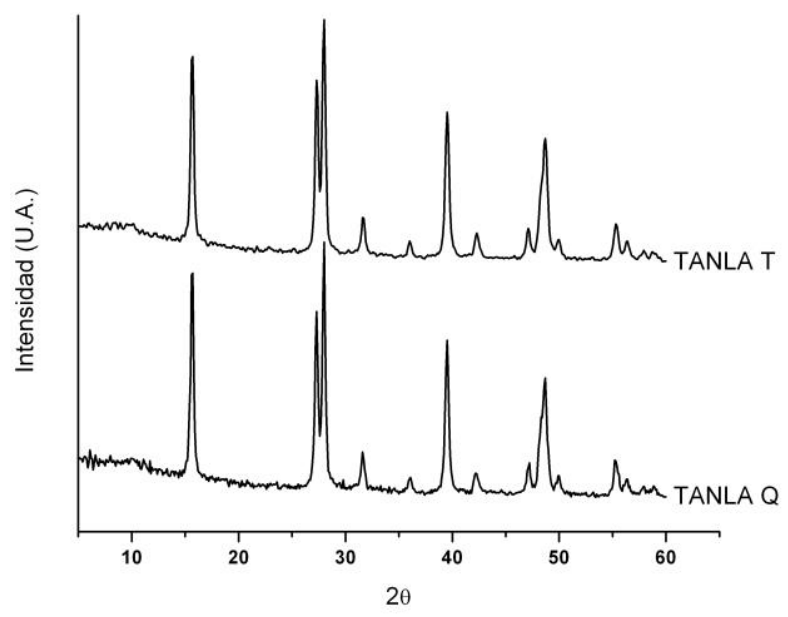

Figura 3: Difractogramas para las muestras TANLA Q y TANLA T calcinadas.

Los difractogramas (Figura 3 ) presentan los mismos picos para ambas muestras, con máximos a $2 \theta$ $=27,93 ; 15,66 ; 27,22 ; 39,49$ y 48,60 respectivamente. Utilizando la base de datos de Joint Committee for Powder Diffraction Sources se concluyó que éstos se corresponden con $\mathrm{La}(\mathrm{OH})_{3}$ (JCPDF\# 36-1481). Conocidas la masas de los "tanatos" calcinadas y los volúmenes de la solución inicial de $\mathrm{La}\left(\mathrm{NO}_{3}\right)_{3} \times 6 \mathrm{H}_{2} \mathrm{O}$ utilizada durante la síntesis, se puede decir que en el pigmento se encuentra el $80 \%$ del La introducido en la preparación.

La cantidad de taninos solubles, a la temperatura de trabajo, se determinó utilizando el reactivo Folin-Denis, que genera un producto coloreado cuando reacciona con monofenoles, polifenoles, flavonoides, taninos y cualquier otra sustancia fácilmente oxidable. El reactivo fue preparado en el laboratorio a partir de $\mathrm{Na}_{2} \mathrm{WO}_{4} \times 2 \mathrm{H}_{2} \mathrm{O}, \mathrm{H}_{3}\left(\mathrm{P}\left(\mathrm{Mo}_{3} \mathrm{O}_{10}\right)_{4}\right) \times \mathrm{H}_{2} \mathrm{O}, \mathrm{H}_{3} \mathrm{PO}_{4}$ (conc.) y agua destilada, calentando a reflujo durante 2 h. La curva de calibración se realizó utilizando ácido tánico como patrón (Figura 4) y las determinaciones se realizaron mediante un espectrómetro UV-VIS [13].

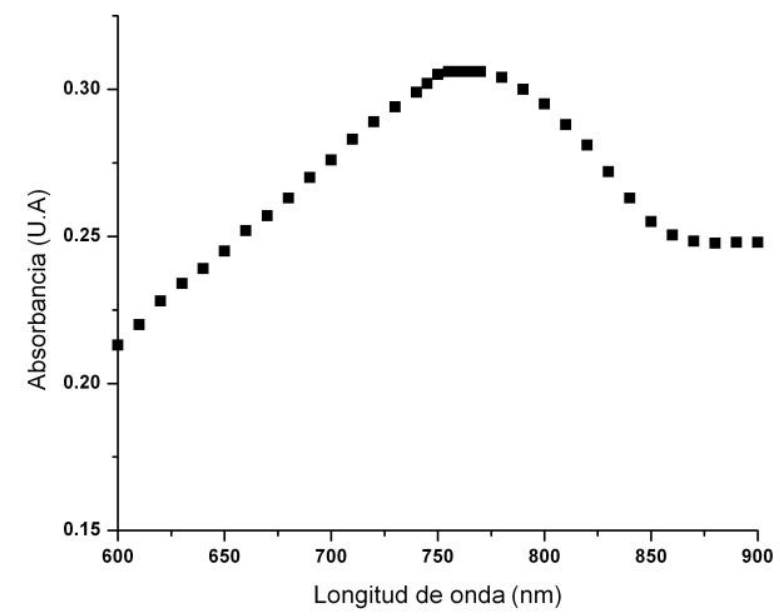

Figura 4: a) Espectro de absorción del ác. tánico. 


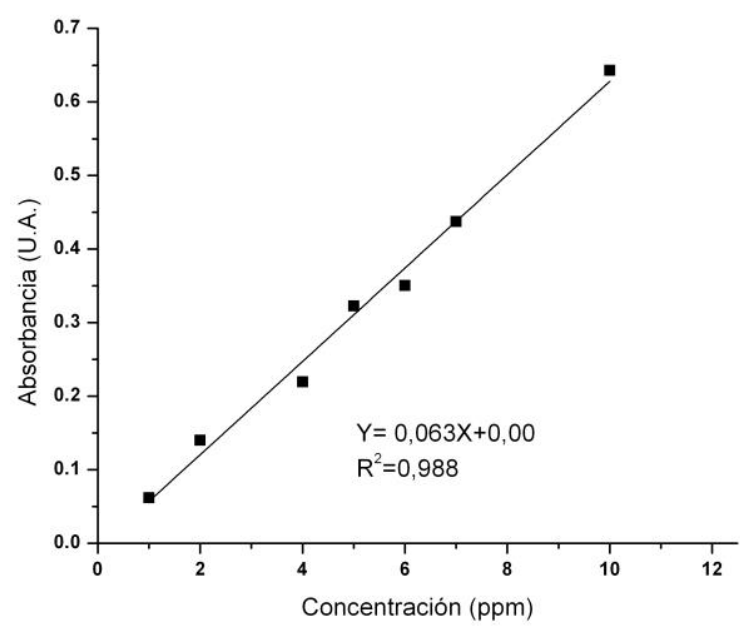

Figura 4: b) curva de calibración.

La cantidad de material oxidable presente en solución en equilibrio fue de 5 ppm para TANLA Q y despreciable para TANLA T.

La concentración de lantano soluble se determinó por una técnica gravimétrica precipitando el lantano como oxalato [14]. En ninguno de los casos se observó la aparición de precipitado, lo que indica que la concentración de lantano estaría por debajo de $1,1 \times 10^{-6} \mathrm{M}$, teniendo en cuenta que el $\mathrm{K}_{\mathrm{PS}}$ del oxalato de lantano es $2,0 \times 10^{-28} \mathrm{M}[15]$.

$$
\mathrm{K}_{\mathrm{PS}}=\left[\mathrm{La}^{+3}\right]^{2} \times[\text { oxalato }]^{3}
$$

\subsection{Estudio de la capacidad inhibidora de corrosión de los pigmentos}

El potencial de corrosión en función del tiempo otorga información respecto a la tendencia a la corrosión que presenta el sustrato (Figura 5).

Los potenciales de corrosión de electrodos de acero SAE 1010 en contacto con soluciones de TQ de 7, 12 y 16 ppm respectivamente, mostraron durante los primeros 150 minutos de inmersión, un desplazamiento del potencial hacia valores más positivos respecto de electrodos similares en la solución de $\mathrm{NaCl}$ 0,025M al incrementarse la concentración de tanino. Hacia el final del ensayo, los Ecorr de las soluciones con 7 y 12 ppm de TQ se acercaron al valor del blanco mientras que para la solución restante se midieron potenciales más negativos (Figura 5 a).

Los potenciales de corrosión en soluciones TT para las distintas concentraciones ensayadas (2, 5 y $10 \mathrm{ppm}$ ) se mantienen alrededor de $-600 \mathrm{mV}$ durante todo el experimento independientemente de la concentración de tanino (Figura 5 b).

El potencial de corrosión de acero en contacto con soluciones de $\mathrm{La}^{+3} 1 \times 10^{-7} \mathrm{M}, 1 \times 10^{-5} \mathrm{M}$ y $1 \times 10^{-3}$ $\mathrm{M}$ respectivamente, varió al comienzo del ensayo entre aproximadamente -400 y $-300 \mathrm{mV}$ siendo más positivo para soluciones más diluidas. Hacia el final del ensayo, hay una variación de similar magnitud, pero la solución más concentrada mostró los valores más positivos indicando una mejor protección de sustrato (Figura $5 \mathrm{c}$ ).

Los potenciales de corrosión de electrodos de acero SAE 1010 en contacto con suspensiones de TANLA Q y TANLA T (1 g en $100 \mathrm{ml})$ se mantuvieron oscilando alrededor de un valor cercano a aprox. $-250 \mathrm{mV}$ para TANLA Q y a aprox. $-225 \mathrm{mV}$ para TANLA $\mathrm{T}$ durante todo el ensayo (240 minutos). 


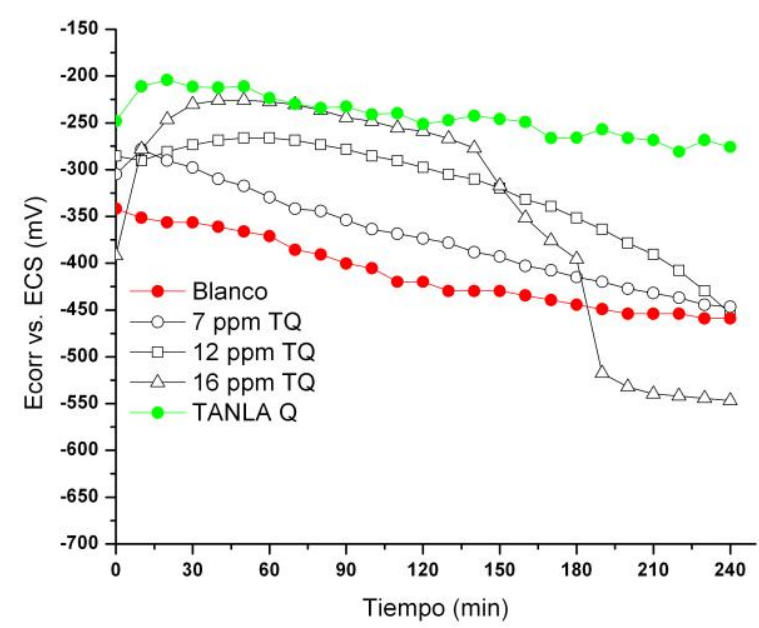

Figura 5: a) Ecorr (vs ECS) en función del tiempo en suspensiones de TQ y TANLA Q.

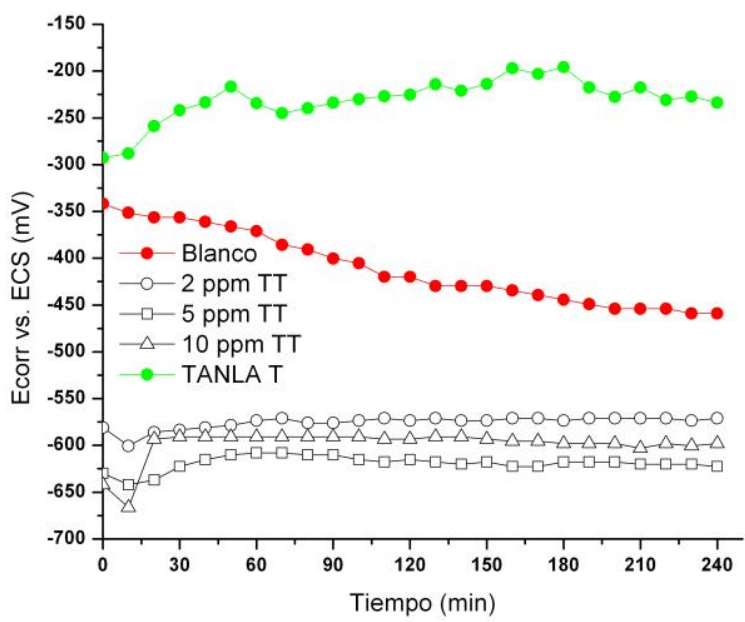

Figura 5: b) Ecorr (vs ECS) en función del tiempo en suspensiones de TT y TANLA T.

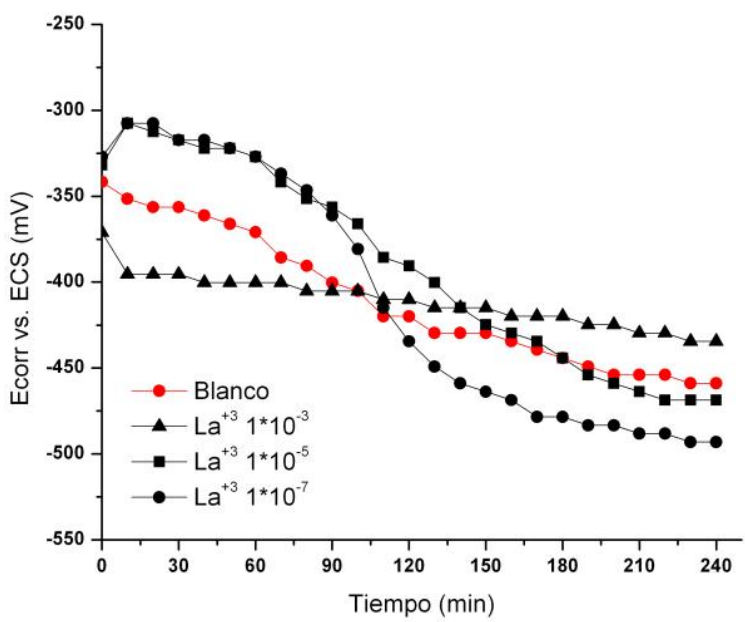

Figura 5: c) Ecorr (vs ECS) en función del tiempo en soluciones de La (III).

La velocidad de corrosión da idea de la rapidez a la que ocurre el ataque electroquímico del sustrato metálico (Tabla 1). 
Luego de 24 h de inmersión, la tendencia a la corrosión del acero SAE1010 se incrementa con la concentración de TT, llegando en algunos casos a superar al blanco. Excepto para la concentración más baja, Icorr aumentó con el tiempo de exposición. Las soluciones TQ presentan un comportamiento donde la velocidad de corrosión del acero alcanzó un mínimo para la concentración intermedia de TQ. Icorr decreció al aumentar el tiempo de ensayo, lo que reveló una mayor capacidad protectora del TQ con respecto al TT. Finalmente, las soluciones de $\mathrm{La}^{+3}$ evidencian su efecto inhibidor al aumentar la concentración de catión.

En la Figura 6 se muestran las curvas de polarización lineal obtenidas.

Si se define la eficiencia de inhibición (EI \%) como [4]:

$\mathrm{EI} \%=\left[\left(\right.\right.$ Icorr $_{0}$-Icorr $) /$ Icorr $\left._{0}\right] \times 100$

siendo Icorr $_{0}$ la velocidad de corrosión del acero inmerso en la solución blanco e Icorr la correspondiente en suspensión de pigmento, la EI del TANLA Q y TANLA T, luego de 24 h de inmersión, fueron del $94 \%$ y $95 \%$ respectivamente. En cambio, los componentes solubles de ambos pigmentos mostraron una EI \% menor, ya sea taninos TT y TQ de en promedio $83,5 \%$, en el mejor de los casos y $\mathrm{La}^{+3} 36 \%$.

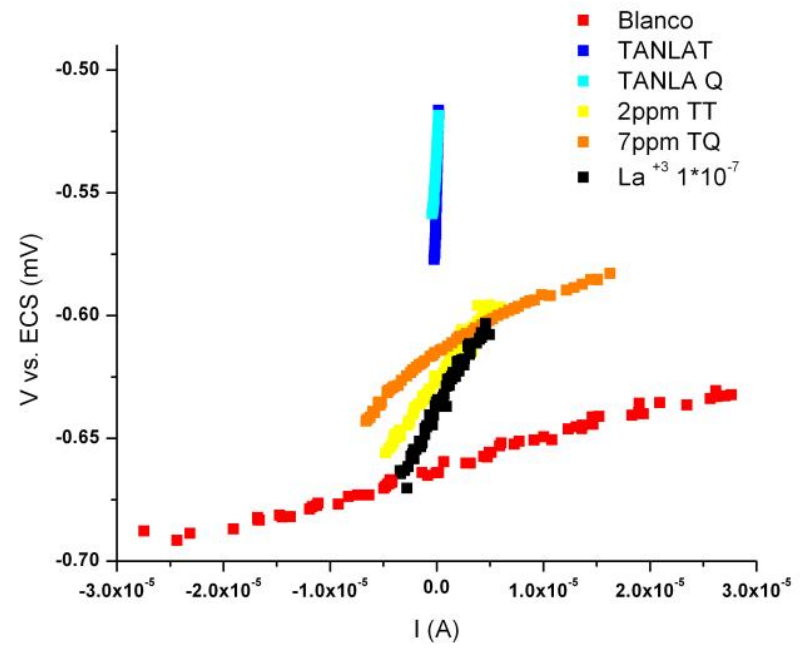

Figura 6: Curvas de polarización lineal de acero en las diferentes soluciones luego de 24 h de inmersión

Tabla 1: Velocidad de corrosión del acero SAE 1010 en $\mathrm{NaCl}$ 0,1 M para distintas concentraciones de los pigmentos.

\begin{tabular}{|c|c|c|c|c|c|}
\hline \multirow[t]{2}{*}{ PIGMENTO } & \multicolumn{2}{|c|}{ CONCENTRACIÓN } & \multicolumn{3}{|c|}{$\begin{array}{l}\text { VELOCIDAD DE CORROSIÓN } \\
\qquad\left(\mu \mathrm{A} / \mathrm{cm}^{2}\right)\end{array}$} \\
\hline & tanino (ppm) & $\mathrm{La}^{+3}(\mathrm{M})$ & $2 \mathrm{~h}$ & $5 \mathrm{~h}$ & $24 \mathrm{~h}$ \\
\hline \multirow{3}{*}{$\begin{array}{l}\text { Tanino de } \\
\text { Tara }\end{array}$} & 2 & ------- & 45,3 & 40,9 & 16,0 \\
\hline & 6 & ------- & 76,7 & 84,1 & 91,8 \\
\hline & 10 & ------ & 132,0 & 163,0 & 166,7 \\
\hline TANLA T & $<1$ & $<1,1.10^{-6}$ & 10,0 & 8,2 & 5,1 \\
\hline \multirow{3}{*}{$\begin{array}{l}\text { Tanino de } \\
\text { quebracho }\end{array}$} & 7 & ------ & 22,8 & 19,8 & 17,4 \\
\hline & 12 & ------ & 10,2 & 11,3 & 9,1 \\
\hline & 17 & ------- & 33,1 & 29,2 & 15,7 \\
\hline TANLA Q & 5 & $<1,1.10^{-6}$ & 7,9 & 6,4 & 5,8 \\
\hline \multirow{3}{*}{$\begin{array}{l}\text { Solución de } \\
\qquad \mathbf{L a}^{+3}\end{array}$} & ------- & $1.10^{-7}$ & 118,0 & 106,6 & 64,8 \\
\hline & ------- & $1.10^{-5}$ & 68,8 & 80,0 & 53,5 \\
\hline & ------ & $1.10^{-3}$ & 4,0 & 3,3 & 2,6 \\
\hline
\end{tabular}




\begin{tabular}{|l|c|c|c|c|c|}
\hline Blanco & ------ & ----- & 152,6 & 138,5 & 101,5 \\
\hline
\end{tabular}

\subsection{Análisis de la superficie}

Las probetas de acero SAE1010 sumergidas durante $24 \mathrm{~h}$ en $\mathrm{NaCl}$ 0,025M (ensayo "blanco") y en suspensiones de TANLA T y TANLA Q se observaron mediante SEM con la finalidad de caracterizar el film obtenido. La composición de las películas, determinada por EDS se observa en la Tabla 2; en el caso del blanco, la película solo contenía Fe y O.

Tabla 2: Cuantificación EDS para las probetas en contacto con las suspensiones de tanatos.

\begin{tabular}{|c|c|c|}
\hline \% peso & TANLA T & TANLA Q \\
\hline $\mathrm{C}$ & 4,71 & 6,65 \\
\hline $\mathrm{O}$ & 3,32 & 2,21 \\
\hline $\mathrm{La}$ & 7,21 & 1,08 \\
\hline $\mathrm{Fe}$ & 84,76 & 90,05 \\
\hline Total & 100 & 100 \\
\hline
\end{tabular}

Del blanco se puede decir que la superficie resultó atacada por el medio agresivo y se observó la formación de una capa de óxido de hierro (Figura 7). Para TANLA T se evidenció la presencia de una película protectora homogénea que por EDS muestra como principales componentes Fe y La, mientras que para TANLA Q se observaron las líneas de pulido lo que indica la formación de una película más delgada que para TANLA T y por EDS el componente mayoritario es Fe (Figura 7).

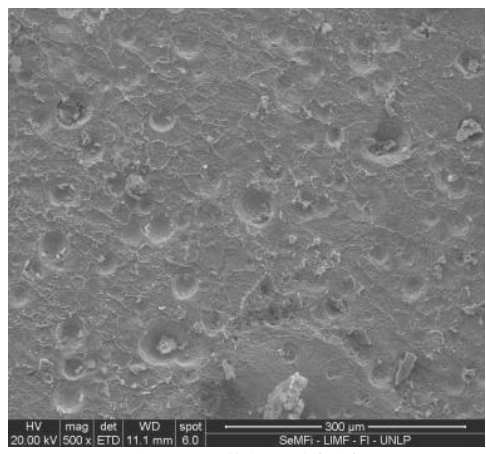

Acero SAE 1010

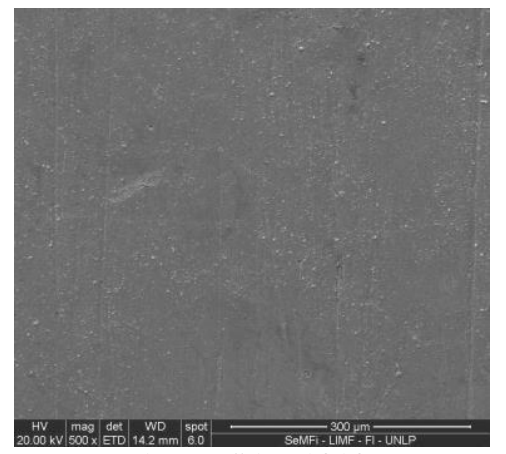

Acero SAE 1010

en contacto con TANLA T

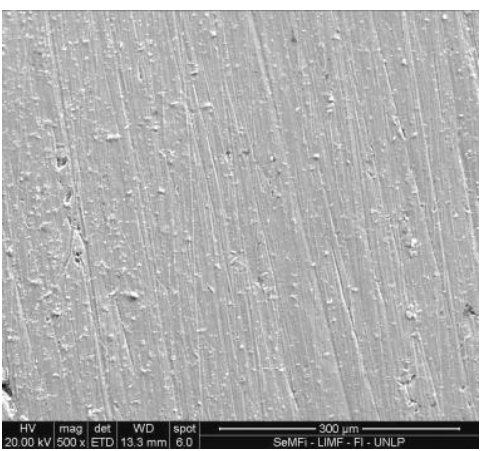

Acero SAE 1010

en contacto con TANLA Q

Figura 7: Microfotografías a 500x de las probetas SAE 1010 en CINa y en contacto con las suspensiones TANLA T y TANLA Q.

\section{CONCLUSIONES}

1. La protección brindada al acero por los "tanatos" de La es mejor que la brindada por los taninos respectivos y mejor que la del ión La(III) aunque la concentración de este último en las suspensiones de "tanato" sea inferior.

2. Teniendo en cuenta los resultados obtenidos, es de preveer que la incorporación de los "tanatos" de La en la formulación de primers otorgue resultados promisorios. 


\section{BIBLIOGRAFIA}

[1] BELlOTTI, N., DEL AMO, B., ROMAGNOLI, R., "Caesalpinia spinosa tannin derivatives for antifouling formulations”; Procedia Materials Science, v. 1, pp. 259-265, 2012.

[2] SANYAL, B., "Organic Compounds as corrosion inhibitors in different environments- A Review"; Progress in Organic Coatings, v. 9, pp. 165-236, Aug. 1981.

[3] MARTINEZ, S., STERN, I., "Thermodynamic characterization of metal dissolution and inhibitor adsorption processes in the low carbon steel/mimosa tannin/sulfuric acid system”,Applied Surface Science, v. 199, n. 1-4, pp. 83-89, Oct. 2002.

[4] RAHIM, A. A., ROCCA, E., STEINMETZ, J., et al., "Mangrove tannins and their flavanoid monomers as alternative steel corrosion inhibitors in acidic medium", Corrosion Science, v. 49, pp. 402-417, Febr. 2007.

[5] BARRERO, C., OCAMPO, L., ARROYAVE, C., "Possible improvements in the action of some rust converters", Corrosion Science, v 43, pp. 1003-1018, 2001.

[6] OCAMPO, L., MARGARIT, I., MATTOS, O., et al., "Performance of rust converter based in phosphoric and tannic acids", Corrosion Science v. 46, pp. 1515-1525, 2004.

[7] ROSELLI, S., REVUELTA, M.V., DEYÁ, M.C., et al., In: Anales SAM/CONAMET: "Pinturas anticorrosivas inteligentes: una alternativa eco-amigable para la protección del acero"; pp. 1-6, Santa Fe, octubre, 2014.

[8] VETERE, V. F., ROMAGNOLI, R., "Chemical and electrochemical assessment of tannins and aqueous primers containing tannins”, Surface Coatings International, v. 81, n. 8, pp. 385-391, Aug. 1998.

[9] ERDEMOĞLU, S.B., GÜÇER, S., "Selective determination of aluminum bound with tannin in tea infusion", Anal. Sci. v. 21, n. 8, pp. 1005-1008, Aug. 2005.

[10] WELCHER, F. J., Organic Analytical Reagents, Vol. II, D. Van Nostrand Company Inc., 1947, New York, pp 61.

[11] GRASEL, F., FLÔRES FERRÃO, M, WOLF, C. R., "Development of methodology for identification the nature of the polyphenolic extracts by FTIR associated with multivariate analysis", Spectrochimica Acta Part A: Molecular and Biomolecular Spectroscopy v. 153, pp. 94-101, Jan. 2016.

[12] SALAVATI-NIASARI, M., HOSSEINZADEH, G., DAVAR, F., "Synthesis of lanthanum hydroxide and lanthanum oxide nanoparticles by sonochemical method", Journal of Alloys and Compounds, v. 509, n. 10, pp. 4098-4103, March. 2011.

[13] SINGLETON, V. L., ROSSI, J. A., Colorimetry of Total Phenolics with PhosphomolybdicPhosphotungstic Acid Reagents, Am J Enol Vitic. v. 16, pp. 144-158, Jan. 1965.

[14] KOLTHOFF, I. M., ELMQUIST, R., "The quantitative determination of lanthanum by precipitation as oxalate or as hydroxide and the higher oxide formation of lanthanum", Journal of the American Chemical Society, v. 53, n. 4, pp 1225-1232, Apr. 1931.

[15] WILSON, C. L., WILSON, D. W., Comprehensive Analytical Chemistry, 1st ed., Amsterdam, Elsevier Publishing Company, 1960. 